\title{
Completeness and incompleteness of the Binet-Legendre metric
}

\author{
Vladimir S. Matveev ${ }^{1}$ • Marc Troyanov ${ }^{2}$
}

Received: 28 August 2014 / Revised: 27 March 2015 / Accepted: 7 April 2015 /

Published online: 2 May 2015

(C) Springer International Publishing AG 2015

\begin{abstract}
The goal of this short paper is to give conditions for the completeness of the Binet-Legendre metric in Finsler geometry. The case of the Funk and Hilbert metrics in a convex domain are discussed.
\end{abstract}

Keywords Finsler metric · Binet-Legendre metric · John ellipsoid · Hilbert geometry · Affine metric

Mathematics Subject Classification $58 \mathrm{~A} 10 \cdot 58 \mathrm{~A} 12 \cdot 53 \mathrm{Cxx}$

\section{Introduction and statement of the main result}

Given a Finsler manifold $(M, F)$, there are several natural ways to construct a Riemannian metric $g$ on the manifold $M$ that is associated to the given Finsler metric. Recently, such constructions were shown to be a useful tool in Finsler geometry, see for example [24-26,34,35].

Remarkably, in most results of all these papers, only the following two properties of the constructions were used:

We thank the Friedrich-Schiller-Universität Jena, EPFL and the Swiss National Science Foundation for their support.

Vladimir S. Matveev

vladimir.matveev@uni-jena.de

Marc Troyanov

marc.troyanov@epfl.ch

1 Institut für Mathematik, Friedrich-Schiller Universität Jena, 07737 Jena, Germany

2 Section de Mathématiques, École Polytechnique Féderale de Lausanne, Station 8, 1015 Lausanne, Switzerland 
- The construction is pointwise: the associated Riemannian metric $g$, restricted to any tangent space of the manifold $M$, depends only on the restriction of the Finsler metric to this tangent space.

- The construction is homogenous: If we multiply the Finsler metric by a conformal factor $\lambda$, the associated Riemannian metric is multiplied by $\lambda^{2}$.

In particular, the proofs of most results in the papers [24-26,35] could be based on any construction of Riemannian metric satisfying the above two conditions, at least when smooth and strictly convex Finsler metrics are considered. Several such constructions have been proposed in recent years. One of the oldest construction seems to be that of Vincze in [36]. The metric in this paper, called the associated Riemannian metric, also satisfies the above requirements, see [36, Remark 3 (ii)]. We refer to $[1,17,37]$ for discussions of further examples.

The construction in [26] is called the Binet-Legendre metric ${ }^{1}$ and has proven to be a flexible and useful tool in Finsler geometry, its definition will be recalled in Sect. 2.2.

Our goal in the present paper is to relate the completeness, or incompleteness, of the Binet-Legendre metric to that of the given Finsler metric. Our main result is in fact the following stronger theorem.

Theorem 1.1 (Main Theorem) Let $(M, F)$ be a continuous Finsler manifold and $g_{\mathrm{BL}}$ be its Binet-Legendre metric, then there exists $C_{1} \in \mathbb{R}$ such that for any $x \in M$ and any $\xi \in T_{x} M$ we have

$$
\sqrt{g_{\text {BL }}(\xi, \xi)} \leq C_{1} \cdot F(x, \xi) .
$$

If the Finsler metric $F$ is quasi-reversible, then there exist constants $C_{2}, C_{3}>0$ such that

$$
C_{2} \cdot F(x, \xi) \leq \sqrt{g_{\mathrm{BL}}(\xi, \xi)} \leq C_{3} \cdot F(x, \xi)
$$

for all $(x, \xi) \in T$ M. In particular $g_{\mathrm{BL}}$ and $F$ are bilipshitz equivalent.

Remarks - Our proof will give explicit (though perhaps not optimal) values for the constants $C_{1}, C_{2}, C_{3}$. The constants $C_{1}$ and $C_{3}$ play the same role, but in the reversible case we have a better constant (namely $C_{3} \leq C_{1} / \sqrt{n}$ ).

- Our theorem implies that if the Binet-Legendre metric associated to a Finsler metric $F$ is complete, then the Finsler metric is also complete, see Corollary 4.2. The converse statement holds in the case of quasi-reversible metric but not in general. We illustrate this phenomenon by an example in Sect. 5.3.

- The quasi-reversibility hypothesis in the second statement is necessary. For instance the Funk metric (discussed below) is forward complete but not backward complete, hence it cannot be bilipschitz equivalent to any Riemannian metric. In fact it is quite clear from Main Theorem that a Finsler metric is bilipschitz to a Riemannian metric if and only if it is quasi-reversible (note the "if" direction follows from Main Theorem, while the "only if" direction is obvious).

\footnotetext{
1 The construction is slightly older and appeared in [12] but its usefulness was overseen until it was reinvented in [26].
} 
- The associated Riemannian metrics constructed in the papers [24,25,35] involve the second derivatives of the given Finlser metric and they generally do not satisfy (1).

The rest of the paper is organized as follows: In Sect. 2 we recall some basic definitions from Finsler geometry and we recall the definition and some basic properties of the Binet-Legendre metric. In Sect. 3 we discuss another auxiliary Riemannian metric, based on the John ellipsoid from convex geometry, and we use it as a tool to prove Theorem 1.1, in Sect. 4, where we also derive some of its simple but important consequences.

In Sect. 5 we discuss some examples. We first recall in Sect. 5.1 the definition of Zermelo metrics in Euclidean domains and in particular the Funk and reverse Funk metrics. In the next Sect. 5.2, we explicitly compute the Binet-Legendre metric associated to a Zermelo metric and in Sect. 5.3 we construct an example of a complete Finsler metric with incomplete Binet-Legendre metric. In Sect. 5.4 we discuss the Hilbert Finsler metric in a convex domain and we use it to compare the Binet-Legendre metric to the so-called affine metric, which is another important Riemannian metric defined in an arbitrary convex domain. In an appendix we show by an example that the Riemannian metric obtained from the John ellipsoid construction may be nonsmooth, even if the initial Finsler metric is smooth.

\section{A brief review of Finsler geometry}

\subsection{Basic definitions: Finsler manifolds, completeness and quasi-reversibility}

A Finsler metric on a smooth manifold $M$ is a continuous function $F: T M \rightarrow[0, \infty)$ such that for every point $x \in M$ the restriction $F_{x}=F_{\mid T_{x} M}$ is a Minkowski norm, that is, for any $\xi, \eta \in T_{x} M$ it satisfies the following properties:

- $F_{x}(\xi)>0$ if $\xi \neq 0$,

- $F_{x}(\xi+\eta) \leq F_{x}(\xi)+F_{x}(\eta)$,

- $F_{x}(\lambda \xi)=\lambda F_{x}(\xi)$ for all $\lambda \geq 0$.

The Finsler metric is said to be c-quasi-reversible, $1 \leq c<\infty$, if $F(x,-\xi) \leq$ $c \cdot F(x, \xi)$ for any $(x, \xi) \in T M$. It is called reversible if it is 1-quasi-reversible, clearly $F$ is reversible if and only if $F_{x}$ is a norm in every tangent space. Classical books introducing to Finsler geometry are $[4,16,23]$; note however that these references assume further restrictions on the Finsler metric $F$, namely that it is smooth on the complement of the zero section of $T M$ and that the vertical Hessian of $F^{2}$ is positive definite. The results in the present paper do not require these conditions and our approach is more similar to that in [8] or [30, Chapter 3].

The distance $d(x, y)$ between two points $x$ and $y$ on a Finsler manifold $(M, F)$ is defined to be the infimum of the length

$$
\ell(\gamma)=\int_{0}^{1} F(\gamma(t), \dot{\gamma}(t)) d t
$$


of all smooth curves $\gamma:[0,1] \rightarrow M$ joining these two points. This distance satisfies the axioms of a metric, except perhaps the symmetry. In fact the condition $d(x, y)=$ $d(y, x)$ is satisfied if and only if the Finsler metric is reversible. Together with the distance comes the notion of completeness: the Finsler manifold $(M, F)$ is said to be forward complete if every forward Cauchy sequence converges. A sequence $\left\{x_{i}\right\} \subseteq M$ is forward Cauchy if for any $\varepsilon>0$, there exists an integer $N$ such that $d\left(x_{i}, x_{i+k}\right)<\varepsilon$ for any $i \geq N$ and $k \geq 0$ (we similarly define backward Cauchy sequences by the condition $d\left(x_{i+k}, x_{i}\right)<\varepsilon$, and the corresponding notion of backward completeness). For a quasi-reversible Finsler metric, forward completeness is evidently equivalent to backward completeness and will simply be called completeness.

A Finsler manifold is equipped with a natural measure: Recall first that a density on the differentiable manifold $M$ is a Borel measure $d v$ such that on any coordinate chart $\phi: U \subset M \rightarrow \mathbb{R}^{n}$, the measure $\phi_{*} d \nu$ is absolutely continuous with respect to the Lebesgue measure, that is it can be written as

$$
\phi_{*} d v=a(x) d x_{1} d x_{2} \ldots d x_{n}
$$

where $x_{1}, x_{2}, \ldots, x_{n}$ are the coordinates defined by the chart $\phi$ and $a(x)$ is a positive measurable function.

A density on the manifold $M$ naturally induces a Lebesgue measure $d \tau_{x}$ on (almost) each tangent space $T_{x} M$, this measure is given by

$$
d \tau_{x}=a(x) d \xi_{1} d \xi_{2} \ldots d \xi_{n}
$$

where $\xi_{1}, \xi_{2}, \ldots, \xi_{n}$ are the natural coordinates on $T_{x} M$ associated to $x_{1}, x_{2}, \ldots, x_{n}$ and $a(x)$ is given by (2).

The Busemann measure $d \mu_{F}$ on the Finsler manifold $\left(M^{n}, F\right)$ is then defined to be the unique density on $M$ such that for every $x \in M$ the volume of the Finsler unit ball $\Omega_{x} \subset T_{x} M$ coincides with the volume of the standard $n$-dimensional Euclidean unit ball, which we denote by $\omega_{n}$. It can be calculated from the formula

$$
d \mu_{F}=\frac{\omega_{n}}{v\left(\Omega_{x}\right)} d \nu
$$

where $d v$ is an arbitrary continuous density on $M$. It is obvious that the Busemann measure $d \mu_{F}$ is independent of the chosen density $d \nu$. It is also clear that in the special case where $F=\sqrt{g}$ for some Riemannian metric $g$, the Busemann measure coincides with the Riemannian volume measure, that is $d \mu_{F}=d \mathrm{vol}_{g}$.

It is also known, but somewhat delicate to prove, that if $F$ is a reversible Finsler metric on $M$, then $d \mu_{F}$ coincides with the $n$-dimensional Hausdorff measure of the metric space associated to the Finsler structure, see [2,8-10].

\subsection{The Binet-Legendre metric}

The Binet-Legendre metric is a canonical Riemannian metric attached to any Finsler metric on a smooth manifold, it has been invented and studied in [12,26]. Let us recall 
the construction: Given a Finsler manifold $(M, F)$ and a point $x$ in $M$, we denote by $\Omega_{x}=\left\{\xi \in T_{x} M: F(x, \xi)<1\right\}$ the $F$-unit tangent ball at $x$. We then define a scalar product on the cotangent space $T_{x}^{*}(M)$ by

$$
g_{\mathrm{BL}}^{*}(\theta, \varphi)=\frac{n+2}{\lambda\left(\Omega_{x}\right)} \int_{\Omega_{x}} \theta(\eta) \cdot \varphi(\eta) d \lambda(\eta),
$$

where $\lambda$ is a Lebesgue measure on $T_{x} M$. Note that this is (up to a constant) the $L^{2}$-scalar product of the linear functions $\theta$ and $\phi$ restricted to $\Omega_{x}$.

Definition 2.1 The Binet-Legendre metric $g_{\mathrm{BL}}$ associated to the Finsler metric $F$ is the Riemannian metric dual to the the scalar product $g_{\mathrm{BL}}^{*}$ defined above on $T_{x}^{*}(M)$.

The Binet-Legendre metric enjoys a number of important properties, let us state in particular the following theorem.

Theorem 2.2 If $(M, F)$ is a Finsler manifold and $g_{\mathrm{BL}}$ is its associated Binet-Legendre metric, then

(a) If $F$ is of class $C^{k}$ on the complement of the zero section of $T M$, then $g_{\mathrm{BL}}$ is a Riemannian metric of class $C^{k}$.

(b) If $\varphi$ is an isometry of $(M, F)$, then it is also an isometry of $\left(M, g_{\mathrm{BL}}\right)$.

(c) If $F_{1}, F_{2}$ are two Finsler metrics on $M$ such that $(1 / \lambda) \cdot F_{1} \leq F_{2} \leq \lambda \cdot F_{1}$ for some function $\lambda: M \rightarrow \mathbb{R}_{+}$, then the corresponding Binet-Legendre metrics satisfy

$$
\frac{1}{\lambda^{2 n}} \cdot g_{\mathrm{BL} 1} \leq g_{\mathrm{BL} 2} \leq \lambda^{2 n} \cdot g_{\mathrm{BL} 1} \cdot
$$

(d) If the Finsler metric $F$ is derived from a Riemannian metric $g$, that is $F=\sqrt{g}$, then $g_{\mathrm{BL}}=g$.

We refer to [26, Theorem 2.4] for the first statement, which is in fact proven for the wider class of partially smooth Finsler metrics. The second statement is obvious and the third and fourth statements are proved in [26, Proposition 12.1].

\section{The John metric on a Finsler manifold}

The proof of Theorem 1.1 will use another auxiliary Riemannian metric, which we call the John metric, that is also associated to a Finsler metric $F$ on the manifold $M$. To explain this metric, recall that any open bounded convex domain $\Omega \subset \mathbb{R}^{n}$ contains a unique ellipsoid of largest volume [18]. This is called the John ellipsoid and we denoted it by $J[\Omega] \subseteq \Omega$. A careful study of the uniqueness proof shows that the John ellipsoid depends continuously on the convex body $\Omega$. If $\Omega$ is symmetric with respect to the origin (that is $-\Omega=\Omega$ ), then $J[\Omega]$ is centered at the origin and we have

$$
J[\Omega] \subseteq \Omega \subseteq \sqrt{n} \cdot J[\Omega]
$$

see [3], [6, p. 214] or [33, Section 3.3]. 
The center of the John ellipsoid is called the John point of $\Omega$ and denoted by $Q_{\Omega}$, we then define the centered John ellipsoid of $\Omega$ as

$$
J_{0}[\Omega]=J[\Omega]-Q_{\Omega} .
$$

It was proved by John in [18, Theorem III], that for an arbitrary open bounded convex set $\Omega \subseteq \mathbb{R}^{n}$, we have

$$
\Omega-Q_{\Omega} \subseteq n \cdot J_{0}[\Omega],
$$

see also [6, p. 210]. Recall that $\Omega$ contains the origin, thus the above inclusion together with the fact that $J_{0}[\Omega]$ is centrally symmetric implies

$$
Q_{\Omega} \in n \cdot J_{0}[\Omega]
$$

It follows from (4) that

$$
\Omega \subseteq Q_{\Omega}+n \cdot J_{0}[\Omega] \subseteq 2 n \cdot J_{0}[\Omega]
$$

The centered John ellipsoid allows us to construct a natural continuous Riemannian metric on any Finsler manifold. More precisely we have the following result.

Proposition 3.1 Any Finsler manifold $(M, F)$ carries a well-defined Riemannian metric $g_{\text {John }}$ of class $C^{0}$ whose unit ball at any point $x \in M$ is the centered John ellipsoid $J_{0}\left[\Omega_{x}\right] \subseteq T_{x} M$ of the Finsler unit ball $\Omega_{x} \subseteq T_{x} M$. Furthermore the following inequality holds:

$$
\frac{1}{2 n} \sqrt{g_{\text {John }}(\xi, \xi)} \leq F(x, \xi)
$$

for any $(x, \xi) \in T M$. If the Finsler metric $F$ is reversible, then we have the better estimates

$$
\frac{1}{\sqrt{n}} \sqrt{g_{\text {John }}(\xi, \xi)} \leq F(x, \xi) \leq \sqrt{g_{\text {John }}(\xi, \xi)}
$$

In particular a reversible Finsler metric $F$ is bilipschitz equivalent to the Riemannian metric $g_{\text {John }}$.

This Riemannian metric $g_{\text {John }}$ will be called the John metric associated to the Finsler metric, it is a natural construction and appeared in the papers [31,32]. Note that the John metric may fail to be $C^{1}$, even if the Finsler metric $F$ is analytic, an example is given in Appendix.

Proof Let $\Omega_{x} \subset T_{x} M$ be the Finsler tangent unit ball at $x \in M$, and let us denote by $J_{0}\left[\Omega_{x}\right] \subseteq T_{x} M$ the corresponding centered John ellipsoid. This ellipsoid is the unit ball of a uniquely defined positive symmetric definite bilinear form on $T_{x} M$. By continuity of the John ellipsoid, these bilinear forms give us a $C^{0}$-Riemannian metric $g_{\text {John }}$ on $M$, that is naturally associated to the Finsler metric $F$.

Inclusion (5) gives us $\Omega_{x} \subset 2 n \cdot J_{0}\left[\Omega_{x}\right]$, which immediately implies inequality (6). In the reversible case, $\Omega_{x} \subseteq T_{x} M$ is symmetric around the origin and from (3) we have the inclusions $J\left[\Omega_{x}\right] \subseteq \Omega_{x} \subseteq \sqrt{n} \cdot J\left[\Omega_{x}\right]$ which are equivalent to (7). The proof of the last assertion is straightforward. 
Our next result says that the volume form of the John metric is comparable to the Busemann measure of the Finsler metric $F$.

Proposition 3.2 Let $(M, F)$ be a Finsler manifold with Busemann measure $\mu_{F}$. Then the following inequalities hold:

$$
d \mu_{F} \leq d \mu_{\mathrm{John}} \leq n^{n} \cdot d \mu_{F}
$$

where $d \mu_{\mathrm{John}}$ is the Riemannian density of the John metric $g_{\mathrm{John}}$ associated to the Finsler metric.

Proof Choose $d v=d \mu_{\text {John }}$ as initial density. Since $J\left[\Omega_{x}\right] \subset \Omega_{x} \subset T_{x} M$ for any point $x$ in $M$, we have

$$
\omega_{n}=\mu_{\mathrm{John}}\left(J_{0}\left[\Omega_{x}\right]\right)=\mu_{\mathrm{John}}\left(J\left[\Omega_{x}\right]\right) \leq \mu_{\mathrm{John}}\left(\Omega_{x}\right) .
$$

Conversely, using (4), we have

$$
\mu_{\text {John }}\left(\Omega_{x}\right)=\mu_{\text {John }}\left(\Omega_{x}-Q_{x}\right) \leq \mu_{\text {John }}\left(n \cdot J_{0}\left[\Omega_{x}\right]\right)=n^{n} \mu_{\text {John }}\left(J_{0}\left[\Omega_{x}\right]\right)=n^{n} \omega_{n},
$$

where $Q_{x}$ is the John point of $\Omega_{x}$. We just proved the inequalities $\omega_{n} \leq \mu_{\text {John }}\left(\Omega_{x}\right) \leq$ $n^{n} \omega_{n}$, which are equivalent to (8).

Remark 3.3 Note that, due to (3), the second inequality in (8) can be improved as follows in the case of a reversible Finsler metric:

$$
\mu_{\mathrm{John}} \leq n^{n / 2} \cdot d \mu_{F}
$$

\section{Proof of Main Theorem and some consequences}

We first prove inequality (1). Recall that by definition the dual of the Binet-Legendre metric $g_{\mathrm{BL}}^{*}$ is given at any point $x \in M$ by the formula

$$
\begin{aligned}
& \int_{\Omega} \theta^{2}(\xi) d \lambda(\xi) \geq \int_{J_{0}[\Omega]+Q_{\Omega}} \theta^{2}(\xi) d \lambda(\xi) \\
& \quad=\int_{J_{0}[\Omega]} \theta^{2}\left(\xi+Q_{\Omega}\right) d \lambda(\xi) \\
& \quad=\int_{J_{0}[\Omega]} \theta^{2}(\xi) d \lambda(\xi)+2 \cdot \theta\left(Q_{\Omega}\right) \int_{J_{0}[\Omega]} \theta(\xi) d \lambda(\xi)+\int_{J_{0}[\Omega]} \theta^{2}\left(Q_{\Omega}\right) d \lambda(\xi) \\
& \quad \geq \int_{J_{0}[\Omega]} \theta^{2}(\xi) d \lambda(\xi) .
\end{aligned}
$$

The last inequality follows from the fact that the ellipsoid $J_{0}[\Omega]$ is centered at the origin, which implies that

$$
\int_{J_{0}[\Omega]} \theta(\xi) d \lambda(\xi)=\theta\left(\int_{J_{0}[\Omega]} \xi d \lambda(\xi)\right)=0 .
$$


On the other hand, inequality (4) implies $\lambda(\Omega) \leq n^{n} \lambda\left(J_{0}[\Omega]\right)$ and we obtain

$$
g_{\mathrm{BL}}^{*}(\theta, \theta)=\frac{n+2}{\lambda(\Omega)} \int_{\Omega} \theta^{2}(\xi) d \lambda(\xi) \geq \frac{n+2}{n^{n} \lambda\left(J_{0}[\Omega]\right)} \int_{J_{0}[\Omega]} \theta^{2}(\xi) d \lambda(\xi) .
$$

Because the ellipsoid $J_{0}[\Omega]$ is the unit ball of the John metric, we have

$$
\frac{n+2}{\lambda\left(J_{0}[\Omega]\right)} \int_{J_{0}[\Omega]} \theta^{2}(\xi) d \lambda(\xi)=g_{\text {John }}^{*}(\theta, \theta) .
$$

Dualizing inequalities (9) and (10), and using (6) from Proposition 3.1, we obtain

$$
\sqrt{g_{\text {BL }}(\xi, \xi)} \leq n^{n / 2} \sqrt{g_{\text {John }}(\xi, \xi)} \leq 2 n^{1+n / 2} F(x, \xi)
$$

as desired.

We now prove the second part of Main Theorem. Assume first that the Finsler metric $F$ is reversible and recall inequalities (7) from Proposition 3.1,

$$
\frac{1}{\sqrt{n}} \sqrt{g_{\text {John }}} \leq F \leq \sqrt{g_{\text {John }}}
$$

Since $g_{\text {John }}$ is Riemannian, it is its own Binet-Legendre metric and we conclude from (12) and Theorem 2.2 (c) that

$$
\frac{1}{n^{n}} g_{\text {John }} \leq g_{\text {BL }} \leq n^{n} g_{\text {John }}
$$

From (12) and (13) we obtain

$$
\frac{1}{n^{n / 2}} F \leq \sqrt{g_{\text {BL }}} \leq n^{(n+1) / 2} F
$$

Assume now more generally that $F$ is $c$-quasi-reversible, that is $F(x,-\xi) \leq$ $c \cdot F(x, \xi)$ for any $(x, \xi) \in T M$. Let us set

$$
F^{\prime}(x, \xi)=\frac{1}{2}(F(x, \xi)+F(x,-\xi))
$$

Clearly, $F^{\prime}$ is reversible and satisfies $2 F^{\prime} /(1+c) \leq F \leq(1+c) F^{\prime} / 2$. If $g_{\mathrm{BL}}^{\prime}$ is the Binet-Legendre metric for $F^{\prime}$, we have from Theorem 2.2 (c) that

$$
\left(\frac{2}{1+c}\right)^{2 n} \cdot g_{\mathrm{BL}}^{\prime} \leq g_{\mathrm{BL}} \leq\left(\frac{1+c}{2}\right)^{2 n} \cdot g_{\mathrm{BL}}^{\prime}
$$

Inequalities (14) applied to the reversible Finsler metric $F^{\prime}$ say that $n^{-n / 2} F^{\prime} \leq \sqrt{g_{\mathrm{BL}}^{\prime}} \leq$ $n^{(n+1) / 2} F^{\prime}$, combining this with (16) we finally obtain 


$$
\sqrt{g_{\mathrm{BL}}} \leq\left(\frac{1+c}{2}\right)^{n} \cdot \sqrt{g_{\mathrm{BL}}^{\prime}} \leq n^{(n+1) / 2}\left(\frac{1+c}{2}\right)^{n} \cdot F^{\prime} \leq n^{(n+1) / 2}\left(\frac{1+c}{2}\right)^{1+n} \cdot F
$$

Similarly

$$
\sqrt{g_{\mathrm{BL}}} \geq\left(\frac{2}{1+c}\right)^{n} \cdot \sqrt{g_{\mathrm{BL}}^{\prime}} \geq \frac{1}{n^{n / 2}}\left(\frac{2}{1+c}\right)^{n} \cdot F^{\prime} \geq \frac{1}{n^{n / 2}}\left(\frac{2}{1+c}\right)^{1+n} \cdot F .
$$

We rewrite the last two inequalities

$$
\frac{1}{n^{n / 2}}\left(\frac{2}{1+c}\right)^{1+n} \cdot F \leq \sqrt{g_{\text {в }}} \leq n^{(n+1) / 2}\left(\frac{1+c}{2}\right)^{1+n} \cdot F .
$$

The theorem is proved.

Remark 4.1 Disregarding the exact constants, we can sketch the proof for the second statement as follows: let us denote by $\mathrm{BL}[F]$ the Binet-Legendre metric of the Finsler metric $F$, by $F^{\prime}$ the (reversible) Finsler metric (15) and by $\sim$ the bilipschitz equivalence. Then, we have consequently shown that

$$
\mathrm{BL}\left[F \mid \sim \operatorname{BL}\left[F^{\prime}\right] \sim \operatorname{BL}\left[g_{\text {John }}^{\prime}\right]=g_{\text {John }}^{\prime} \sim F^{\prime} \sim F .\right.
$$

Let us now state some simple consequences of Main Theorem.

Corollary 4.2 Let $(M, F)$ be an arbitrary Finsler manifold. If the Binet-Legendre metric $g_{\mathrm{BL}}$ is complete, then $F$ is both forward and backward complete.

Proof Let $\left\{x_{j}\right\}$ be a forward Cauchy sequence for the metric $F$, then the first statement from Main Theorem implies that $\left\{x_{j}\right\}$ is a Cauchy sequence for the Riemannian metric $g_{\text {BL }}$. It is therefore a convergent sequence by hypothesis. The proof for a backward Cauchy sequence is the same.

Corollary 4.3 Let $(M, F)$ be a quasi-reversible Finsler manifold, then

(a) The Binet-Legendre metric $g_{\mathrm{BL}}$ is complete if and only if the given Finsler metric $F$ is complete.

(b) The Riemannian volume density of $g_{\mathrm{BL}}$ is comparable to the Busemann density $d \mu_{F}$.

(c) Two quasi-reversible Finsler manifolds are quasi-isometric if and only if the associated Riemannian manifold with their respective Binet-Legendre metrics are quasi-isometrics.

Recall that the Finsler manifolds $\left(M_{1}, F_{1}\right)$ and $\left(M_{2}, F_{2}\right)$ are quasi-isometric if there exists a map $f: M_{1} \rightarrow M_{2}$ and a constant $A$ such that for any $p, q \in M_{1}$ we have $d_{F_{2}}(f(p), f(q)) \leq A \cdot\left(d_{F_{1}}(p, q)+1\right)$ and for any $y \in M_{2}$ there exists $x \in M_{1}$ with $d_{F_{2}}(f(x), y) \leq A$.

Proof The property (a) is an immediate consequence of Main Theorem since completeness is a property which is stable under bilipschitz equivalence. 
Property (b) is also a consequence of Main Theorem: Let us denote by $d \mu_{\mathrm{BL}}$ the Riemannian volume density of $g_{\mathrm{BL}}$. Then the Busemann density is

$$
d \mu_{F}=\frac{\omega_{n}}{\mu_{\mathrm{BL}}\left(\Omega_{x}\right)} d \mu_{\mathrm{BL}}
$$

where $\Omega_{x}$ is the unit ball in $T_{x} M$ for the Finsler metric $F$. Because $g_{\text {в }}$ is bilipschitz equivalent to $F$, we have $(1 / k) \cdot B_{x} \subset \Omega_{x} \subset k \cdot B_{x}$ for some constant $k$ where $B_{x} \subseteq$ $T_{x} M$ is the unit ball for the metric $g_{\mathrm{BL}}$. It follows at once that

$$
\omega_{n} k^{-n} d \mu_{\mathrm{BL}} \leq d \mu_{F} \leq \omega_{n} k^{n} d \mu_{\mathrm{BL}} .
$$

In order to prove (c), recall that quasi-isometry is an equivalence relation among metric spaces, see e.g. [8, Section 8.3], and bilipschitz equivalence is clearly a special case of quasi-isometry. The claim follows thus immediately from Main Theorem.

Remark 4.4 The first inequality in (17) can be improved: it is known that the Riemannian volume is in fact always smaller or equal to the Busemann measure, that is $d \mu_{\mathrm{BL}} \leq d \mu_{F}$, and the equality holds if and only if $F$ is Riemannian. This fact also holds without the reversibility assumption and follows, e.g. from [22, Theorem 1], see also [12, Theorem 3.2].

\section{Examples and applications}

\subsection{Zermelo metrics in a domain}

Let us consider a bounded convex domain $\Omega$ in $\mathbb{R}^{n}$ and a $C^{k}$-map $u: \mathcal{U} \rightarrow \Omega$ where $\mathcal{U} \subset \mathbb{R}^{n}$ is an arbitrary domain (one may, but need not, assume that $\mathcal{U}=\Omega$ ). The Finsler metric $F_{u}$ on $\mathcal{U}$ whose associated tangent unit ball at $x \in \mathcal{U}$ is the domain $\Omega$ centered at $u(x)$ is called the Zermelo metric associated to the map $u: \mathcal{U} \rightarrow \Omega$. Note that in this definition we use the canonical identification $T_{x} \mathcal{U} \equiv \mathbb{R}^{n}$. The Finslerian unit tangent ball at $x \in \mathcal{U}$ is thus given by

$$
\Omega_{x}=\Omega-u(x)=\left\{\xi \in \mathbb{R}^{n}: \xi+u(x) \in \Omega\right\} .
$$

The Finsler metric $F$ is then given by

$$
F_{u}(x, \xi)=\inf \{t>0: \xi \in t(\Omega+u(x))\}=\inf \left\{t>0: \frac{\xi}{t}+u(x) \in \Omega\right\} .
$$

Equivalently, for any $\xi \neq 0$,

$$
F_{u}(x, \xi)>0 \quad \text { and } \quad \frac{\xi}{F_{u}(x, \xi)}+u(x) \in \partial \Omega .
$$

We refer to [5] and [16, Section 1.4] for a discussion of the Zermelo metric and the relation with Zermelo's navigation problem. $^{2}$

\footnotetext{
2 Note that [16] has an opposite sign convention for the vector field $u$.
} 


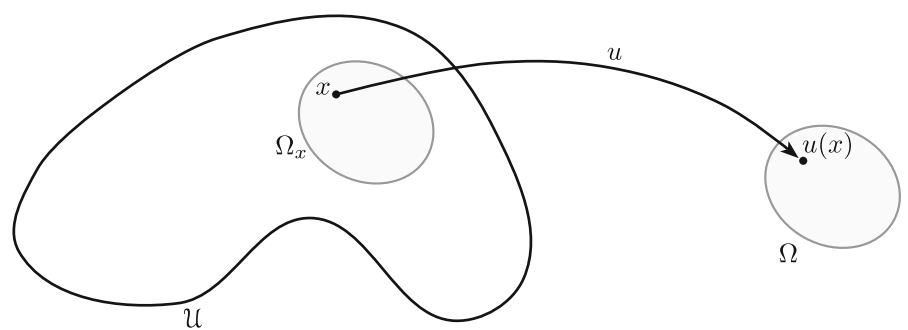

Fig. 1 A Zermelo metric in the domain $\mathcal{U}$, the Finlser unit ball at $x$ is given by $\Omega$ with $u(x)$ as origin

Examples (a) If $u(x)=c \in \Omega$ is constant, then the corresponding Zermelo metric is invariant by translation. It is thus the Minkowski metric whose unit ball is given by $\Omega-c$ (Fig. 1).

(b) If $\mathcal{U}=\Omega$ and $u(x)=x$ is the identity map, then the corresponding Zermelo metric is called the Funk metric and denoted by $F_{\text {Funk }}$. The Finsler unit ball at the point $x \in \Omega$ is the convex domain $\Omega$ itself, but with the point $x$ as its center (this metric is therefore also called the tautological Finsler structure).

(c) The reverse of a Zermelo metric is also a Zermelo metric. Recall that the reverse of a Finsler metric $F$ is the Finsler metric ${ }^{r} F$ given by ${ }^{r} F(x, \xi)=F(x,-\xi)$. In the case of the Zermelo metric $F_{u}$ associated to the map $u: \mathcal{U} \rightarrow \Omega$ we easily check that the reverse metric ${ }^{r} F_{u}$ is the Zermelo metric associated to the map $-u: \mathcal{U} \rightarrow-\Omega$, that is we have the identity

$$
{ }^{r} F_{u}(x, \xi)=F_{-u}(x, \xi)=F_{u}(x,-\xi) .
$$

(d) In particular the reverse of the Funk metric, which is denoted by $F_{\text {RFunk }}$, is the Zermelo metric in $\mathcal{U}=\Omega$ associated to the map $u: \Omega \rightarrow-\Omega$ given by $u(x)=-x$. The Finsler unit ball is the symmetric image of $\Omega$ with respect to the center of symmetry at $x$.

We refer to [16], [30, Chapters 2 and 3] for some background on Funk and reverse Funk geometry. In particular, the following formula for the distance is well known: if $p$ and $q$ are distinct points in $\Omega$ and $a, b$ are the two points lying on the intersection of the line through $p$ and $q$ with the boundary $\partial \Omega$ and if $a, q, p, b$ appear in that order on that line then

$$
d_{\text {Funk }}(p, q)=\log \frac{|a-p|}{|a-q|} \quad \text { and } \quad d_{\text {RFunk }}(p, q)=\log \frac{|b-q|}{|b-p|} .
$$

The following facts are classical.

Proposition 5.1 Let $\Omega \subset \mathbb{R}^{n}$ be a bounded convex domain, then

- The reverse Funk metric satisfies $F_{\mathrm{RF} u n k}(x, \xi)=F_{\mathrm{Funk}}(x,-\xi)$. They are both invariant under affine transformations preserving $\Omega$.

- The Funk metric in $\Omega$ is forward complete but not backward complete. The reverse Funk metric is backward complete and not forward complete. 
- Both metrics are projective, meaning that the Euclidean straight lines are geodesics.

- If the bounded convex domain $\Omega \subset \mathbb{R}^{n}$ has a boundary of class $C^{k}$, then $F_{\text {Funk }}$ and $F_{\mathrm{RFunk}}$ are also of class $C^{k}$ (on the complement of the zero section).

The first statement is obvious and second and third statements are proved in [16] for domains with smooth and strongly convex domains and in [30, Chapters 2 and 3]. The last statement follows from the implicit function theorem.

\subsection{Computation of the Binet-Legendre metric for a Zermelo metric}

Since the Funk metric is not backward complete, it follows from Corollary 4.2 that its associated Binet-Legendre metric is incomplete. In this section we provide another proof for the incompleteness. More generally we compute the Binet-Legendre metric for a general Zermelo metric in a domain $\mathcal{U}$ and show that it is never complete unless $\mathcal{U}=\mathbb{R}^{n}$.

We will in fact consider a more general situation. A Borel probability $\mu$ measure on $\mathbb{R}^{n}$ is said to have finite quadratic moment if

$$
\int_{\mathbb{R}^{n}}|x|^{2} d \mu(x)<\infty
$$

where $|x|$ is the Euclidean norm. The probability $\mu$ is said to be affinely nondegenerate if for any nonzero linear form $\varphi: \mathbb{R}^{n} \rightarrow \mathbb{R}$ and any point $a$ in $\mathbb{R}^{n}$ we have

$$
\int_{\mathbb{R}^{n}} \varphi^{2}(x-a) d \mu(x)>0 .
$$

Equivalently the support of the measure $\mu$ is not contained in an affine hyperplane. Given such a measure $\mu$ satisfying (19) and (20), we associate to any $C^{k}$-smooth function $u: \mathcal{U} \rightarrow \mathbb{R}^{n}$ the following scalar product on $\left(\mathbb{R}^{n}\right)^{*}$ :

$$
g_{x}^{*}(\theta, \varphi)=\gamma \int_{\mathbb{R}^{n}} \theta(\zeta-u(x)) \cdot \varphi(\zeta-u(x)) d \mu(\zeta)
$$

where the constant $\gamma>0$ is an arbitrary parameter. If the measure is centered at the origin, that is $\int \zeta d \mu(\zeta)=0$, then we have

$$
\begin{aligned}
& g_{x}^{*}(\theta, \varphi)=\gamma\left\{\int_{\mathbb{R}^{n}} \theta(\zeta) \varphi(\zeta) d \mu(\zeta)-\theta(u(x)) \int_{\mathbb{R}^{n}} \varphi(\zeta) d \mu(\zeta)\right. \\
&\left.-\varphi(u(x)) \int_{\mathbb{R}^{n}} \theta(\zeta) d \mu(\zeta)+\theta(u(x)) \varphi(u(x))\right\} \\
&= \gamma \int_{\mathbb{R}^{n}} \theta(\zeta) \varphi(\zeta) d \mu(\zeta)+\gamma \cdot \theta(u(x)) \varphi(u(x)) \\
&= g_{0}^{*}(\theta, \varphi)+\gamma \cdot \theta(u(x)) \varphi(u(x)) .
\end{aligned}
$$


In the second equality we have used $\int \varphi(\zeta) d \mu(\zeta)=\varphi\left(\int \zeta d \mu(\zeta)\right)=0$. If we furthermore assume that the coordinates are chosen to be orthonormal for the metric $g_{0}$ at the origin, then the coefficients of $g_{x}^{*}$ are

$$
g^{i j}=g_{x}^{*}\left(\varepsilon^{i}, \varepsilon^{j}\right)=\delta^{i j}+\gamma u_{i} u_{j}
$$

where $\varepsilon^{1}, \ldots, \varepsilon^{n}$ is the dual canonical basis. Inverting this matrix, one obtains the following Riemannian metric on $\mathbb{R}^{n}$ :

$$
g_{i j}=\delta_{i j}-\frac{\gamma u_{i} u_{j}}{1+\gamma|u(x)|^{2}}
$$

The Binet-Legendre metric for the Zermelo metric corresponding to the function $u: \mathcal{U} \rightarrow \Omega$, where $\Omega$ is a bounded convex domain, is the special case of this construction corresponding to the constant $\gamma=n-2$ and the measure $d \mu=\chi_{\Omega} / \operatorname{Vol}(\Omega) d x$. Indeed, by definition of the Zermelo metric, the Finsler tangent ball at a point $x \in \mathcal{U}$ is given by

$$
\Omega_{x}=\left\{\xi \in T_{x} \mathcal{U}: F(x, \xi)<1\right\}=\left\{\xi \in \mathbb{R}^{n}: \xi \in(\Omega-u(x))\right\}=\Omega-u(x)
$$

(here we use the canonical identification $T_{x} \mathcal{U}=\mathbb{R}^{n}$ ). The dual Binet-Legendre metric associated to the Zermelo metric is then given by

$$
g_{x}^{*}(\theta, \varphi)=\frac{n+2}{\operatorname{Vol}\left(\Omega_{x}\right)} \int_{\Omega_{x}} \theta(\xi) \varphi(\xi) d \xi=\frac{n+2}{\operatorname{Vol}\left(\Omega_{x}\right)} \int_{\Omega} \theta(\zeta-u(x)) \varphi(\zeta-u(x)) d \zeta
$$

It follows that in an appropriate coordinate system, the Binet-Legendre associated to a Zermelo metric in a domain $\mathcal{U}$ is given by (22) with $\gamma=n+2$.

Observe in particular that since $u(x)$ belongs to the bounded domain $\Omega$ for any $x \in \mathcal{U}$, the tensors (21) and (22) are always bounded. This implies in particular that the Binet-Legendre metric of a Zermelo metric in a domain $\mathcal{U}$ is bilipschitz equivalent to the Euclidean metric. In particular it is complete if and only if $\mathcal{U}=\mathbb{R}^{n}$.

Remark 5.2 In the special case of the Funk or reverse Funk metric, we have $u(x)=$ $\pm x$. It follows from (22) that for any bounded convex domain $\Omega \subset \mathbb{R}^{n}$, the BinetLegendre metric is given in some coordinate system by

$$
g_{i j}=\delta_{i j}-\frac{\gamma x_{i} x_{j}}{1+\gamma|x|^{2}}
$$

Observe that this formula is independent of the geometry of $\Omega$.

Remark 5.3 The previous construction of a metric associated to a probability measure in $\mathbb{R}^{n}$ is natural in multivariate statistics. Let $X_{1}, \ldots, X_{n}$ be random variables and assume that the random vector $X=\left(X_{1}, \ldots, X_{n}\right)$ is nondegenerate. Recall that this means that there are no constants $a_{1}, \ldots, a_{n}$ and $C$ such that $\operatorname{Prob}\left(\sum_{i} a_{i} X_{i}=C\right)=1$. Assume also that the random vector $X$ has finite second moments, that is $\mathbb{E}\left(X_{i}^{2}\right)<\infty$, 
$1 \leq i \leq n$, where $\mathbb{E}(\cdot)$ is the expectation. The joint distribution of those variables is the probability measure $\mu$ on $\mathbb{R}^{n}$ defined by $\mu(B)=\operatorname{Prob}(X \in B)$ for any Borel set $B \subset \mathbb{R}^{n}$, and under the given hypothesis the measure $\mu$ satisfies the previous conditions (19) and (20). Choosing the function $u(x)=x$, the corresponding metric $g_{x}^{i j}$ in $\left(\mathbb{R}^{n}\right)^{*}$ is then the matrix of product moments,

$$
g_{x}^{i j}=\mathbb{E}\left(\left(X_{i}-x_{i}\right)\left(X_{j}-x_{j}\right)\right)
$$

At the barycenter of $\mu$, this matrix is the covariance matrix of the random vector $X$ and is often denoted by $\Sigma$. The inverse matrix $g_{i j}$ is called the precision or concentration matrix. In the case of Gaussian random variables, this matrix is related to conditional independencies between the random variables.

\subsection{An example of a complete metric with incomplete Binet-Legendre metric}

In this subsection we briefly give an example of a Finsler metric that is both forward and backward complete and whose associated Binet-Legendre metric is incomplete, showing that the converse to Corollary 4.2 fails.

The example is given by a Zermelo metric that interpolates between the Funk metric (which is forward complete) and the reverse Funk metric (which is backward complete). It can be built in any bounded convex domain, but we will only describe it in the standard unit ball $\mathbb{B}^{n} \subset \mathbb{R}^{n}$.

Using (18), we see that the Funk distance in $\mathbb{B}^{n}$ from the origin to a point $x \in \mathbb{B}^{n}$ is given by

$$
d_{\text {Funk }}(0, x)=\log \frac{1}{1-|x|},
$$

therefore the open ball of radius $t$ centered at the origin for the Funk metric is given by

$$
W_{t}=\left\{x \in \mathbb{R}^{n}:|x|<1-\mathrm{e}^{-t}\right\} .
$$

Let us now choose a smooth function $u: \mathbb{B}^{n} \rightarrow \mathbb{B}^{n}$ such that for any integer $k \in \mathbb{N}$ we have

$$
u(x)= \begin{cases}x & \text { if } \quad x \in W_{4 k+1} \backslash W_{4 k}, \\ -x & \text { if } \quad x \in W_{4 k+3} \backslash W_{4 k+2}\end{cases}
$$

The Zermelo metric $F_{u}$ associated to the function $u$, coincides with the Funk metric in $W_{4 k+1} \backslash W_{4 k}$ and to the reverse Funk metric in $W_{4 k+3} \backslash W_{4 k+2}$ for any integer $k \in \mathbb{N}$. In particular we have

$$
x \notin W_{4 k+3} \quad \Longrightarrow \quad d_{u}(0, x) \geq k \text { and } d_{u}(x, 0) \geq k .
$$


Fig. 2 The distance in the Funk or reverse Funk metric is given by the logarithm of the ratio of the Euclidean distances to the boundary

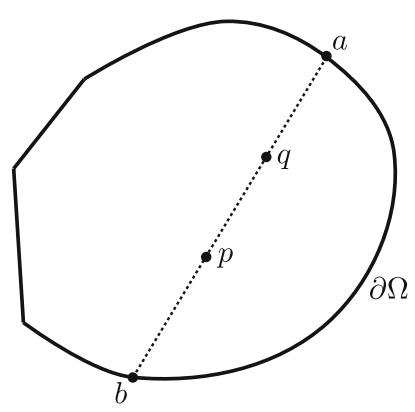

Because $W_{4 k+3}$ is relatively compact in $\mathbb{B}^{n}$, it is clear that the metric $F_{u}$ is both forward and backward complete. Since we proved in the previous subsection that the associated Binet-Legendre metric is not complete, we have produced an example of a complete Finsler metric with incomplete Binet-Legendre metric.

\subsection{The Hilbert metric and the "affine metric" in a bounded convex domain}

The symmetrization of the Funk metric in a bounded convex domain $\Omega$ is called the Hilbert metric in that domain, the Finsler norm is thus given by

$$
F_{\text {Hill }}(x, \xi)=\frac{1}{2}\left(F_{\text {Funk }}(p, \xi)+F_{\text {Funk }}(p,-\xi)\right) \text {. }
$$

Referring to the notations in Fig. 2, we have the following formula for the distance between two points $p$ and $q$ :

$$
d_{\text {Hilb }}(p, q)=\frac{1}{2}\left(d_{\text {Funk }}(p, q)+d_{\text {Funk }}(q, p)\right)=\frac{1}{2} \log \left(\frac{|a-p|}{|a-q|} \cdot \frac{|b-q|}{|b-p|}\right) .
$$

We refer to the books [11,29] for a short introduction to Hilbert geometry and to [30] for an overview of some recent developments.

We have the following result about the Binet-Legendre metric associated to the Hilbert metric.

Proposition 5.4 The Binet-Legendre metric associated to the Hilbert metric in a bounded convex domain $\Omega$ is a complete Riemannian metric. This metric is invariant under the group of projective transformations preserving the domain and it is bilipschitz equivalent to the Hilbert metric.

Proof The Hilbert metric is clearly reversible and it is not difficult to check from formula (23) that it is complete. The second statement in Theorem 1.1 implies that its associated Binet-Legendre metric is bilipshitz equivalent to the Hilbert metric, in particular it is also complete.

The Hilbert metric is invariant under projective transformations since the distance is expressed in terms of the cross ratio of four aligned points. Using Theorem 2.2 (b), 
we deduce that the Binet-Legendre metric is also invariant under projective transformations.

Another important projectively invariant metric in a convex domain can be constructed from the solution to some Monge-Ampère equation. It is based on the following statement.

Theorem 5.5 Let $\mathcal{U} \subset \mathbb{R}^{n}$ be an arbitrary bounded convex domain. Then there exists a unique solution to the following Monge-Ampère equation:

$$
\operatorname{det} \frac{\partial^{2} u}{\partial x_{i} \partial x_{j}}=\left(-\frac{1}{u}\right)^{n+2},
$$

which is smooth, positive, strictly concave, continuous in the closure $\overline{\mathcal{U}}$ and vanishes on the boundary $\partial \mathrm{U}$.

This theorem was first proved in 1974 by Loewner and Nirenberg for the case of smooth, 2-dimensional strictly convex domain [19] and in 1977 by Cheng and Yau for the general case $[13,19,21]$.

Definition 5.6 The affine metric on the convex domain $\mathcal{U}$ is the Riemannian metric defined as

$$
g_{\mathrm{Aff}}=-\frac{1}{u} \sum_{i, j} \frac{\partial^{2} u}{\partial x_{i} \partial x_{j}} d x_{i} d x_{j}
$$

where $u: \mathcal{U} \rightarrow \mathbb{R}^{n}$ is the above solution to (24).

Observe that by the strict concavity of $u$, the metric $g_{\text {Aff }}$ is positive definite, hence Riemannian. The name "affine metric" has been proposed in relation to the Blaschke theory of affine hypersurfaces, see [7,20,28]. The affine metric enjoys the following properties.

Theorem 5.7 - The affine metric $g_{\text {Aff }}$ is complete and invariant under projective transformations leaving the domain $\mathcal{U}$ invariant.

- The affine metric $g_{\text {Aff }}$ is bilipschitz equivalent to the Hilbert metric: there exists a constant c such that

$$
\frac{1}{c} \cdot F_{\text {Hilb }} \leq \sqrt{g_{\text {Aff }}} \leq c \cdot F_{\text {Hilb }} .
$$

A proof of the first statement is given in [19, Sections 6 and 9], see also [14,15]. The second statement is a recent result by Benoist and Hulin [7, Proposition 3.4]. Observe that the completeness of $g_{\text {Aff }}$ also follows from the second statement, since the Hilbert metric is complete. From the previous theorem and Theorem 1.1 we then have

Corollary 5.8 The Binet-Legendre metric $g_{\mathrm{BL}}$ associated to the Hilbert metric in a properly convex domain $\mathcal{U} \subset \mathbb{R P}^{n}$ is bilipschitz equivalent to the affine metric $g_{\text {Aff. }}$. 
In conclusion, both the Binet-Legendre and the affine metric in a convex domain are complete, invariant under projective transformation and bilipschitz equivalent to the Hilbert metric. Observe however that the construction of the affine metric is based on hard analysis to solve a nonlinear elliptic partial differential equation, so even the existence of such a metric is a nontrivial fact. On the other hand, the Binet-Legendre metric is based on a direct and quite elementary geometric construction. This metric can be effectively computed, at least for sufficiently simple domains, see e.g. [27].

Acknowledgments The authors are thankful to Rolf Schneider for useful discussions.

\section{Appendix 1: Non-smoothness of the John metric}

The John metric, like the Binet-Legendre metric, is a natural construction in Finsler geometry that enjoys good functorial properties, in particular properties (b), (c) and (d) of Theorem 2.2 also hold for the John metric. However, the John metric is in general not smooth and this fact creates serious limits to its potential usefulness in Finsler geometry. We illustrate this phenomenon by the following example. Consider the following Finsler metric $F$ on $M=\mathbb{R}^{n}$ :

$$
F(x, \xi)=\|\xi\|_{p(x)}=\left(\sum_{i=1}^{n}\left|\xi_{i}\right|^{p(x)}\right)^{1 / p(x)}
$$

where $p$ is the function $p(x)=1+e^{x_{1}}$. If one identifies $T_{x} M$ with $\mathbb{R}^{n}$, the Finsler unit ball is

$$
\Omega_{x}=\left\{\xi \in \mathbb{R}^{n}: \sum_{i=1}^{n}\left|\xi_{i}\right|^{p(x)}<1\right\}
$$

It is easy to see that the John ellipsoid of $\Omega_{x}$ is an Euclidean ball centered at the origin. Indeed, each $\Omega_{x}$ is invariant with respect to the symmetries $\sigma_{i}:\left(\ldots, \xi_{i}, \ldots\right) \mapsto$ $\left(\ldots,-\xi_{i}, \ldots\right)$ and $\sigma_{i j}:\left(\ldots, \xi_{i}, \ldots, \xi_{j}, \ldots\right) \mapsto\left(\ldots, \xi_{j} \ldots, \xi_{i}, \ldots\right)$, and since the John ellipsoid $J\left[\Omega_{x}\right]$ of $\Omega_{x}$ is unique, it must be $\sigma_{i}$ - and $\sigma_{i j}$-invariant for all $i, j=$ $1, \ldots, n$. Since the Euclidean balls centered at the origin are the only ellipsoid invariant with respect to all such symmetries, the John ellipsoid must be such a ball. The radius $r$ of the ball $J\left[\Omega_{x}\right]$ only depends on $p=p(x)$ and a calculation shows that

$$
r(x)=\min \left\{1, n^{1 / 2-1 / p}\right\}= \begin{cases}n^{1 / 2-1 / p} & \text { if } 1<p \leq 2 \\ 1 & \text { if } p \geq 2\end{cases}
$$

Indeed, for $p \leq 2$ a common point of the boundary of $J\left[\Omega_{x}\right]$ of $\Omega_{x}$ is given by $\xi=(1,0, \ldots, 0)$, while for $p \geq 2$ a common point of the boundary of $J\left[\Omega_{x}\right]$ of $\Omega_{x}$ is $\xi=\left((1 / n)^{1 / p}, \ldots,(1 / n)^{1 / p}\right)$ (Fig. 3$)$.

It is elementary to check that the function $r(x)$ is not differentiable when $x_{1}=\log 2$, that is $p=2$. Therefore the ellipsoid $J\left[\Omega_{x}\right]$ does not depend smoothly on $x$. Moreover, 


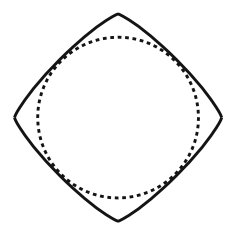

$p=1.2$

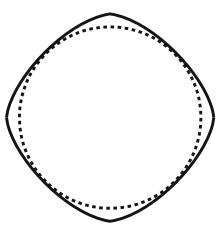

$p=1.5$

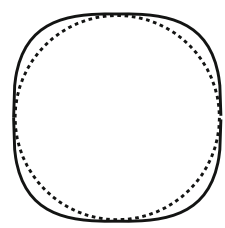

$p=3$

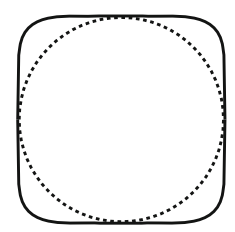

$p=6$

Fig. 3 The convex bodies $\Omega_{x}$ and their John ellipsoids

the metric $g_{\text {John }}$ has a discontinuous curvature and therefore cannot be made smooth by a $C^{0}$-change of coordinates. We have thus constructed an analytical Finsler metric $F$ on $\mathbb{R}^{n}$ such that the associated John metric is given at the point $x$ by

$$
g_{\text {John }}(\xi, \eta)=\frac{1}{r^{2}(x)}\langle\xi, \eta\rangle,
$$

where $r(x)$ is not differentiable.

\section{Appendix 2: About the constant $C_{1}$}

Recall inequality (11):

$$
\sqrt{g_{\text {BL }}(\xi, \xi)} \leq 2 n^{1+n / 2} F(x, \xi)
$$

this gives the estimates $C_{1}=2 n^{1+n / 2}$ in Theorem 1.1. However, arguing as in [18], one can slightly improve inequality (5) as follows:

$$
\Omega \subseteq \sqrt{2 n(n+1)} \cdot J_{0}[\Omega]
$$

Inequality (6) can thus be improved as

$$
\frac{1}{\sqrt{2 n(n+1)}} \sqrt{g_{\text {John }}(\xi, \xi)} \leq F(x, \xi) .
$$

Using (25), inequality (11) can now be improved to

$$
\sqrt{g_{\text {BL }}(\xi, \xi)} \leq \sqrt{2 n(n+1)} n^{n / 2} F(\xi)
$$

That is we have the better estimate $C_{1}=\sqrt{2 n(n+1)} n^{n / 2}$ in Theorem 1.1 .

\section{References}

1. Aikou, T.: Averaged Riemannian metrics and connections with application to locally conformal Berwald manifolds. Publ. Math. Debrecen 81(1-2), 179-198 (2012) 
2. Álvarez Paiva, J.C., Thompson, A.C.: Volumes on normed and Finsler spaces. In: Bao, D., Bryant, R.L., Chern, S.-S., Shen, Z. (eds.) A Sampler of Riemann-Finsler Geometry. Mathematical Sciences Research Institute Publications, vol. 50, pp. 1-48. Cambridge University Press, Cambridge (2004)

3. Ball, K.: An elementary introduction to modern convex geometry. In: Levy, S. (ed.) Flavors of Geometry. Mathematical Sciences Research Institute Publications, vol. 31, pp. 1-58. Cambridge University Press, Cambridge (1997)

4. Bao, D., Chern, S.-S., Shen, Z.: An Introduction to Riemann-Finsler Geometry. Graduate Texts in Mathematics, vol. 200. Springer, New York (2000)

5. Bao, D., Robles, C., Shen, Z.: Zermelo navigation on Riemannian manifolds. J. Differential Geom. 66(3), 377-435 (2004)

6. Barvinok, A.: A Course in Convexity. Graduate Studies in Mathematics, vol. 54. American Mathematical Society, Providence (2002)

7. Benoist, Y., Hulin, D.: Cubic differentials and finite volume convex projective surfaces. Geom. Topol. 17(1), 595-620 (2013)

8. Burago, D., Burago, Yu., Ivanov, S.: A Course in Metric Geometry. Graduate Studies in Mathematics, vol. 33. American Mathematical Society, Providence (2001)

9. Busemann, H.: Intrinsic area. Ann. Math. 48(2), 234-267 (1947)

10. Busemann, H.: The geometry of Finsler spaces. Bull. Amer. Math. Soc. 56(1), 5-16 (1950)

11. Busemann, H.: The Geometry of Geodesics. Dover Books on Mathematics. Dover Publications, Mineola (2005)

12. Centore, P.: Volume forms in Finsler spaces. Houston J. Math. 25(4), 625-640 (1999)

13. Cheng, S.-Y., Yau, S.-T.: On the regularity of the Monge-Ampère equation $\operatorname{det}\left(\partial^{2} u / \partial x_{i} \partial x_{j}\right)=$ $F(x, u)$. Comm. Pure Appl. Math. 30(1), 41-68 (1977)

14. Cheng, S.-Y., Yau, S.-T.: The real Monge-Ampère equation and affine flat structures. In: Chern, S.-S., Wu, W. (eds.) Proceedings of the 1980 Beijing Symposium on Differential Geometry and Differential Equations, vols. 1-3 (Beijing, 1980), pp. 339-370. Science Press, Beijing (1982)

15. Cheng, S.-Y., Yau, S.-T.: Complete affine hypersurfaces. Part 1. The completeness of affine metrics. Comm. Pure Appl. Math. 39(6), 839-866 (1986)

16. Chern, S.-S., Shen, Z.: Riemann-Finsler Geometry. Nankai Tracts in Mathematics, vol. 6. World Scientific, Hackensack (2005)

17. Crampin, M.: On the construction of Riemannian metrics for Berwald spaces by averaging. Houston J. Math. 40(3), 737-750 (2014)

18. John, F.: Extremum problems with inequalities as subsidiary conditions. In: Studies and Essays Presented to R. Courant on his 60th Birthday, January 8, 1948, pp. 187-204. Interscience, New York (1948)

19. Loewner, C., Nirenberg, L.: Partial differential equations invariant under conformal or projective transformations. In: Ahlfors, L.V., Kra, I., Maskit, B., Nirenberg, L. (eds.) Contributions to Analysis, pp. 245-272. Academic Press, New York (1974)

20. Loftin, J.: Survey on affine spheres. In: Li, L. (ed.) Handbook of Geometric Analysis, No. 2. Advanced Lectures in Mathematics, vol. 13, pp. 161-191. International Press, Somerville (2010)

21. Loftin, J., Wang, X.-J., Yang, D.: Cheng and Yau's work on the Monge-Ampère equation and affine geometry. Geometry and Analysis, No. 1. Advanced Lectures in Mathematics, vol. 17, pp. 163-179. International Press, Somerville (2011)

22. Lutwak, E., Yang, D., Zhang, G.: A new ellipsoid associated with convex bodies. Duke Math. J. 104(3), 375-390 (2000)

23. Matsumoto, M.: Foundations of Finsler Geometry and Special Finsler Spaces. Kaiseisha Press, Shigaken (1986)

24. Matveev, V.S.: Riemannian metrics having common geodesics with Berwald metrics. Publ. Math. Debrecen 74(3-4), 405-416 (2009)

25. Matveev, V.S., Rademacher, H.-B., Troyanov, M., Zeghib, A.: Finsler conformal Lichnerowicz-Obata conjecture. Ann. Inst. Fourier (Grenoble) 59(3), 937-949 (2009)

26. Matveev, V.S., Troyanov, M.: The Binet-Legendre metric in Finsler geometry. Geom. Topol. 16(4), 2135-2170 (2012)

27. Metzner, G.S.: Binet-Legendre Metrik für Hilbert-Metriken und deren Glattheit. Bachelorarbeit, University of Jena (2013)

28. Nomizu, K., Sasaki, T.: Affine Differential Geometry. Cambridge Tracts in Mathematics, vol. 111. Cambridge University Press, Cambridge (1994) 
29. Papadopoulos, A.: Metric Spaces, Convexity and Nonpositive Curvature. IRMA Lectures in Mathematics and Theoretical Physics, vol. 6. European Mathematical Society, Zürich (2005)

30. Papadopoulos, A., Troyanov, M. (eds.): Handbook of Hilbert Geometry. IRMA Lectures in Mathematics and Theoretical Physics, vol. 22. European Mathematical Society, Zürich (2014)

31. Planche, P.: Géométrie de Finsler sur les Espaces Symétriques. PhD thesis, Université de Genève (1995)

32. Planche, P.: Structures de Finsler invariantes sur les espaces symétriques. C. R. Acad. Sci. Paris Sér. I Math. 321(11), 1455-1458 (1995)

33. Thompson, A.C.: Minkowski Geometry. Encyclopedia of Mathematics and Its Applications, vol. 63. Cambridge University Press, Cambridge (1996)

34. Torromé, R.G.: Average structures associated with a Finsler space (2005). arXiv:math/050105

35. Vincze, Cs: A new proof of Szabó's theorem on the Riemann-metrizability of Berwald manifolds. Acta Math. Acad. Paedagog. Nyházi. 21(2), 199-204 (2005)

36. Vincze, Cs: On a scale function for testing the conformality of a Finsler manifold to a Berwald manifold. J. Geom. Phys. 54(4), 454-475 (2005)

37. Vincze, Cs: Average methods and their applications in differential geometry I. J. Geom. Phys. 92, 194-209 (2015) 